\title{
Myc : le paradoxe d'un oncogène induisant la mort cellulaire programmée
}

S'il est des gènes connus et documentés pour favoriser la division cellulaire, ce sont bien les oncogènes. La situation semble en fait plus compliquée si l'on se réfère aux récents travaux du groupe de Gérard Evan à Londres [1]. Ceux-ci soulèvent en effet l'idée provocante que le proto-oncogène $c-m y c$, l'un des gènes les plus étudiés, contrôlerait à la fois la prolifération et la mort cellulaire programmée, soit deux phénomènes qui sont pour le moins antagonistes.

Le proto-oncogène $c$-myc est l'homologue cellulaire d'oncogènes viraux portés par différents virus aviaires et félin capables d'induire des leucémies et des sarcomes. Il code pour un facteur de transcription, dont l'un des partenaires vient d'être caractérisé $\left(\mathrm{m} / \mathrm{s} n^{\circ} 8\right.$, vol. 7, p. 865 et 866). Bien que certaines données soient contestées, il est peut-être aussi un effecteur de la réplication. Sa régulation est extrêmement fine et fait intervenir de nombreuses régulations transcriptionnelles, posttranscriptionnelles et post-traductionnelles lui permettant de réprimer efficacement son activité en quelques dizaines de minutes [2]. Cette observation suggère déjà, si l'on est finaliste, que sa surexpression accidentelle doit avoir des conséquences importantes sur la physiologie cellulaire.

De nombreux travaux ont établi l'implication de $c-m y c$ dans le contrôle de la prolifération cellulaire. Plus particulièrement, il est connu pour être l'un des gènes les plus rapidement induits en réponse à l'addition de facteurs de croissance. Bien qu'il soit exprimé à un niveau significatif tout au long du cycle cellulaire, tant au niveau de son ARN messager que de sa protéine, le retrait des mitogènes entraîne l'effondrement de son expression, cela quelle que soit la phase du cycle. Il en est de même lors de différents modèles in vitro pour autant que celle-ci soit associée à l'arrêt de la division. Inversement, son expression ectopique dans les mêmes cellules favorise la prolifération au détriment de la différenciation. De plus, son inactivation fonctionnelle par des oligonucléotides ou des ARN antisens dirigés contre son ARN messager bloque la division cellulaire in vitro.

Sa capacité à participer au développement tumoral est solidement démontrée. Tout d'abord, l'altération de sa régulation par des mécanismes variés aboutit à une expression élevée dans la plupart des cancers naturels (pour revue, voir [3]). Bien évidemment cela suggère, mais ne démontre pas, la responsabilité de cet oncogène dans la progression tumorale. Cette dernière idée est expérimentalement démontrée par le fait que le gène $c-m y c$, lorsqu'il est modifié de manière appropriée, est capable de conférer une certaine indépendance vis-à-vis du sérum à différents types de cellules primaires dans des tests de transformation in vitro et d'induire des tumeurs variées dans des souris transgéniques ou infectées par des rétrovirus recombinants $[2,3]$. De plus, il est capable de renforcer le pouvoir tumorigène d'autres oncogènes dans les mêmes tests in vitro et les souris transgéniques. Cependant, et malgré de nombreuses études, il n'a pas encore été établi si l'effet tumorigène est lié à la simple surexpression de l'oncogène ou à une dérégulation qui empêcherait son inactivation à des périodes critiques du cycle cellulaire. Dans ce contexte, les récents travaux d'Evan et al. sont particulièrement troublants. Reprenant des expériences déjà largement documentées, ces auteurs ont été intrigués par l'observation que certaines lignées de fibroblastes de rat exprimant constitutivement un gène $c-m y c$ transfecté s'arrêtent, au moins en apparence et en opposition avec tous les rapports publiés, de croître en présence de faibles concentrations de sérum. En fait, des numérations précises couplées à l'étude de la néosynthèse de l'ADN génomique ont montré que deux phénomènes, s'équilibrant parfois, coexistent dans les cultures étudiées : la prolifération et la mort, apparemment gouvernées de manière aléatoire, d'une fraction notable de la population de cellules. Une analyse fouillée a montré que la lyse cellulaire est strictement dépendante de l'activité et de l'abondance de la protéine c-Myc. En effet, des molécules chimères à activité conditionnelle couplant $\mathrm{Myc}$ au récepteur aux œstrogènes (Myc-ER) nécessitent la présence absolue de l'activatcur spécifique (le $\beta$-œstradiol) pour reproduire l'observation initiale. D'autre part, la fréquence de lyse est proportionnelle au niveau d'expression intracellulaire de Myc lorsque les expériences sont conduites avec une protéine normale transfectée. Ces résultats sont importants puisqu'ils réconcilient toutes les données de la littérature et expliquent qu'avec des niveaux intermédiaires d'expression, les autres investigations menées aient seulement permis de mettre en évidence la perte de dépendance vis-à-vis du sérum induite par myc. L'étude cinémicroscopique a révélé que la fragmentation cellulaire est initiée par la perte des contacts intercellulaires et intervient après une trentaine de minutes en présentant les signes diagnostiques de la mort programmée par apoptose : condensation nucléaire, nécrose membranaire, condensation cytoplasmique et fragmentation cellulaire $[4,5]$. De plus, en utilisant différents procédés de synchronisation, les auteurs ont montré que l'induction de l'apoptose par les protéines Myc-ER est efficace à toute phase du cycle, et même dans les cellules quiescentes. Enfin, l'analyse de différents mutants 
fonctionnels a révélé que ce sont les mêmes domaines structuraux qui sont impliqués à la fois dans la mort cellulaire et toutes les autres fonctions connues du gène : régulation transcriptionnelle, co-transformation avec d'autres oncogènes, auto-répression du gène par son propre produit et inhibition de certaines voies de différenciation. Couplée au fait que l'induction de la lyse cellulaire est rapide, cette observation est compatible avec l'idée que la protéine c-Myc induirait directement des gènes " apoptotiques" spécifiques. Certains d'entre eux étant déjà clonés [6], cette hypothèse devrait pouvoir être testée rapidement.

En résumé, les travaux d'Evan et ses collaborateurs ont permis d'établir qu'en l'absence de mitogènes, la forte expression de c-myc entraîne la mort cellulaire programmée. De façon intéressante, cette observation permet d'introduire l'idée novatrice que ce gène, s'il est bien capable d'induire la prolifération cellulaire en présence de stimulation adéquate, possède aussi de façon intrinsèque la capacité de protéger les individus du développement tumoral puisque sa dérégulation quantitative en absence de stimulation par des facteurs de croissance doit entraîner la mort des cellules mutées. Cette proposition va bien sûr à l'encontre, d'une part, de tous les modèles de carcinogenèse actuels qui font intervenir des régulations positives et négatives dues à des entités moléculaires différentes*, et, d'autre part, des nombreuses observations impliquant myc dans le développement tumoral. En fait, le paradoxe n'est qu'apparent et plusieurs scenarios non mutuellement exclusifs peuvent être proposés pour prendre en compte la diversité des situations. Dans le premier, les cellules sont stimulées à proliférer par des facteurs externes. Cela impose un "dialogue" avec le stroma. Cette notion n'est pas nouvelle et est bien illustrée par les situations inflammatoires qui accompagnent, voire initient, le développement de certaines tumeurs

\footnotetext{
- Cependant, des résultals très récents indiquent que des cellules transfectées avec un gène ras activé et stimulées par les esters de phorbol sont égalemenl induites à s'engager dans un processus apoplotique $\left(\mathrm{m} / \mathrm{s} \mathrm{n}^{\circ} 5\right.$, vol. 8 , p. 505). De méme l'hyperexpression in vivo de l'antigène $T d u$ virus SV 40 dans les cônes de la rétine de souris transgéniques provoque leur mort $\left(\mathrm{m} / \mathrm{s} \mathrm{n}^{\circ} 6\right.$, vol. 8 , p. 613)

\section{TIRÉS A PART}

M. Piechaczyk.

par le biais d'une production localisée de cytokines. En absence d'activation mitogène, la situation est plus subtile. Ainsi, le phénotype tumoral pourrait se révéler au travers d'une surexpression relativement modérée, suffisante pour favoriser la croissance mais insuffisante pour induire la mort cellulaire. Certains lymphomes de Burkitt ont depuis longtemps intrigué les oncologistes par les faibles quantités d'ARN et de protéine produits à partir des gènes $c-m y c$ activés par translocation. Ils pourraient représenter un exemple de cette situation. Par ailleurs, des mutations secondaires altérant les voies de transduction des signaux mitogènes ou le processus apoptotique sont envisageables. Pour ce qui concerne la première possibilité, la capacité de $c-m y c$ à coopérer dans la transformation maligne avec les gènes ras mutés, qui codent pour des petites protéines $G$ [7-9], est peut-être une des illustrations les plus frappantes. D'autre part, le proto-oncogène $b c 12$, dont la forme oncogène est connue pour coopérer avec myc dans le développement de tumeurs lymphocytaires B, est capable de bloquer la mort cellulaire programmée dans les tissus lymphoïdes ([10] $\mathrm{m} / \mathrm{s} n^{\circ} 1$, vol. 7, p. 88 et $n^{\circ} 4$, vol. 8, p. 392).

Les conclusions du groupe anglais apportent donc un plus conceptuel au champ déjà fertile de la prolifération cellulaire. Gageons qu'elles ouvrent par là-même un domaine d'investigation actif où les biologistes cellulaires et moléculaires, au-delà de l'élucidation des mécanismes liant myc à l'apoptose, auront à déterminer si l'observation est généralisable à tous les types cellulaires et à d'autres proto-oncogènes

\section{RÉFÉRENCES}

1. Evan GI, Wyllie AH, Gilbert CS, et al. Induction of apoptosis in fibroblasts by c-myc protein. Cell 1992 ; 69 : 119-28.

2. Spencer C, Groudine M. Control of $c-m y c$ gene in normal and ncoplastic cells. Adv Cancer Res 1991 ; 56 : 1-48.

3. Field JK, Spandidos DA. The role of ras and myc oncogenes in human solid tumors and their relevance in diagnosis and prognosis. Anticancer Res $1990 ; 10: 1-22$.

4. Bursoh W, Kleine L, Tenniswood M. The biochemistry of cell death by apoptosis. Biochem Cell Biol 1990 ; 68 : 1071-4.

5. Goldstein P. Mort programmée et terrain cellulaire. médecine/sciences $1991 ; 7: 681$

6. Owens G, Hahn W, Cohen J. Identification of mRNAs associated with programmed cell death in immature thymocytes. Mol Cell Biol 1991 ; 11 : 4177-88.

7. De Gunzburg J. Les petites protéines $G$ médecine/sciences $1992 ; 4: 322$

8. Goud B. Transport vésiculaire des cellules eucaryotes et petites protéines G. médecine/sciences $1992 ; 4: 326-33$.

9. Kahn A. Les protéines Ras et GAP, des relais sur la voie de transmission du signal passant par les tyrosines kinases. médecine/sciences $1992 ; 8: 471-5$

10. Hockenbery D, Nunez G, Milliman C, et al. Bcl-2 is an inner mitochondrial membrane protein that blocks programmed cell death. Nature 1990 ; 348 : 334-6.

\section{Marc Piechaczyk}

Laboratoire de biologie moléculaire URA Cnrs 1191, génétique moléculaire, place $E$. Bataillon, 34095 Montpellier Cedex 05, France.

\section{ERRATA}

$\mathrm{m} / \mathrm{s} n^{\circ}$ 5, vol. 8, mai 1992

- page 506, dans la référence [2] de la brève: "L'oncogène ras : signal mitogène ou signal de mort ?, il faut lire : Mol Carcinogenesis et non pas Carcinogenesis.

- page 428, les clichés de la figure 2 de l'article de P. Nicolas et al. ont été obtenus par C. Hernandez et F. Dagger (Universidad Central de Venezuela, Caracas). 